\title{
Nonrelativistic supersymmetry in noncommutative space
}

\author{
Gustavo S. Lozano ${ }^{\mathrm{a}, *, 1}$, Olivier Piguet ${ }^{\mathrm{b}}$, Fidel A. Schaposnik ${ }^{\mathrm{c}, 2}$, Lucas Sourrouille ${ }^{\mathrm{a}}$ \\ a Departamento de Física, FCEyN, Universidad de Buenos Aires Pab. 1, Ciudad Universitaria, Buenos Aires, Argentina \\ ${ }^{\mathrm{b}}$ Universidade Federal do Espirito Santo, UFES, Vitória, ES, Brazil \\ ${ }^{c}$ Departamento de Física, Facultad de Ciencias Exactas, Universidad Nacional de La Plata, C.C. 67, 1900 La Plata, Argentina
}

Received 3 August 2005; accepted 23 September 2005

Available online 10 October 2005

Editor: L. Alvarez-Gaumé

\begin{abstract}
We analyze a model of nonrelativistic matter in $(2+1)$-dimensional noncommutative space. The matter fields interact with gauge fields whose dynamics is dictated by a Chern-Simons term. We show that it is possible to choose the coupling constants in such a way that the model has and extended supersymmetry and Bogomolnyi equations can be found.
\end{abstract}

(c) 2005 Elsevier B.V. All rights reserved.

In the past few years, field theories defined in noncommutative (NC) space have received much attention mainly in connection with the effective low energy description of string theories [1]. For the particular case of $(2+1)$ dimensional space, it has also been argued that Chern-Simons theories in NC space can be used as an effective description of the physics of the quantum Hall effect $[2,3]$.

Motivated by these facts, the extension to NC space of the Jackiw and Pi model (JP) [4] of nonrelativistic matter interacting with gauge fields whose dynamics is governed by Chern-Simons fields was first considered in [5].

In ordinary space, this model, which is related to the physics of the Aharonov-Bohm problem, provides a nontrivial example of a gauge theory invariant under the action of the Galilean group [6]. Indeed, the space-time invariance group is larger as the theory is also invariant under dilations and conformal transformations, at least at the classical level. As it is the case for many gauge theories, the scale invariance of the action is broken by quantum corrections. Interestingly, the invariance is recovered for a particular relation of coupling constants [7].

\footnotetext{
* Corresponding author.

E-mail addresses: lozano@df.uba.ar (G.S. Lozano), opiguet@yahoo.com (O. Piguet), fidelschaposnik@yahoo.co.uk (F.A. Schaposnik), 1sourrouille@yahoo.es (L. Sourrouille).

1 Associated with CONICET.

2 Associated with CICBA.
} 
In NC space, the model looses some of the symmetries present in ordinary space. This is somewhat expected as noncommutativity breaks explicitly the scale symmetry and the boost sector of the Galilean group (for a detailed discussion see [8]). Despite this fact, both versions of the model share some important aspects, like the existence of BPS equations for a particular relation of coupling constant $[4,5,9]$.

In ordinary space, the JP model admits and $N=2$ supersymmetric extension [10], providing another example of the by know well established connection between BPS equations and supersymmetry [11-14]. This case is particularly interesting as it provides a nontrivial explicit realization of the graded Galilean symmetry originally discussed in [15].

In this Letter we shall examine possibility of building a supersymmetric extension of the JP model in noncommuative space. We shall show that indeed it is possible to do so precisely for the same relation of coupling constants for which BPS equation exist.

We will be interested in $d=2+1$ noncommutative space characterized by the relations

$$
\left[x_{1}, x_{2}\right]=i \theta, \quad\left[x_{i}, t\right]=0,
$$

where $\theta$ is a real constant with dimension of length squared. It will be convenient to introduce complex variables $z$ and $\bar{z}$

$$
z=\frac{1}{\sqrt{2}}\left(x^{1}+i x^{2}\right), \quad \bar{z}=\frac{1}{\sqrt{2}}\left(x^{1}-i x^{2}\right),
$$

which can be related to annihilation and creation operators $\hat{a}$ and $\hat{a}^{\dagger}$ acting on a Fock space,

$$
\hat{a}=\frac{1}{\sqrt{\theta}} z, \quad \hat{a}^{\dagger}=\frac{1}{\sqrt{\theta}} \bar{z},
$$

so that (1) becomes

$$
\left[\hat{a}, \hat{a}^{\dagger}\right]=1 \text {. }
$$

In this way, through the action of $a^{\dagger}$ on the vacuum state $|0\rangle$, eigenstates of the number operator

$$
\hat{N}=a^{\dagger} a
$$

are generated. With our conventions, derivatives in the Fock space are given by

$$
\partial_{z}=-\frac{1}{\sqrt{\theta}}\left[\hat{a}^{\dagger},\right], \quad \partial_{\bar{z}}=\frac{1}{\sqrt{\theta}}[\hat{a},],
$$

and integration on the noncommutative plane should be interpreted as a trace

$$
\int d^{2} x \rightarrow 2 \pi \theta \operatorname{Tr}
$$

We are interested in the model of nonrelativistic matter interacting with gauge fields whose dynamics is governed by the Chern-Simons term that has been considered in [5]. The action associated to this model can be written as

$$
S=S_{\mathrm{cs}}+\int\left(i \phi^{\dagger} D_{t} \phi-\frac{1}{2 m}\left(D_{i} \phi\right)^{\dagger} D_{i} \phi+\lambda_{1}\left(\phi^{\dagger} \phi \phi^{\dagger} \phi\right)\right) d^{3} x .
$$

Here, $S_{\mathrm{cs}}$ is the Chern-Simon action ${ }^{3}$

$$
S_{\mathrm{cs}}=\int d^{3} x\left(-\frac{\kappa}{4 c i}\left[\left(\partial_{t} A\right)_{+} A_{-}-\left(\partial_{t} A\right)_{-} A_{+}\right]-A_{0} \kappa B_{12}\right),
$$

\footnotetext{
3 Throughout this Letter we will be using the notation $V^{ \pm}=V^{1} \pm i V^{2}$ where $V^{1}$ and $V^{2}$ are the components of any vector $V$ in the plane.
} 
where

$$
B_{12}=\frac{1}{2 i}\left(\partial_{-} A_{+}-\partial_{+} A_{-}\right)-\frac{e}{2 c}\left[A_{-}, A_{+}\right],
$$

and $\phi$ denotes a complex bosonic field. The interaction with the gauge fields is introduced via the covariant derivatives

$$
D_{t} \phi=\partial_{t} \phi+i e A_{0} \phi, \quad D_{i} \phi=\partial_{i} \phi-i e A_{i} \phi .
$$

The model is invariant under gauge transformations,

$$
\phi^{\prime}=U \phi, \quad \psi^{\prime}=U \psi, \quad A_{i}=U^{-1} A_{i} U-\frac{i}{e} \partial_{i} U U^{-1} .
$$

The model described by the action (8) is the realization in NC space of the one originally discussed in [4]. Notice that in the $\mathrm{NC}$ case one needs to choose a particular ordering in the covariant derivative. For definiteness, we will be working with the "fundamental" representation (11)-(12) but the other cases can be handled similarly.

In order to explore the possibility of building a supersymmetric extension of the model, we enlarge the field content of the theory and include a nonrelativistic fermion $\psi$. The action of the model then becomes

$$
\begin{aligned}
S= & S_{\mathrm{cs}}+\int d^{3} x\left(i \phi^{\dagger} D_{t} \phi+i \psi^{\dagger} D_{t} \psi-\frac{1}{2 m}\left(D_{i} \phi\right)^{\dagger} D_{i} \phi-\frac{1}{2 m}\left(D_{i} \psi\right)^{\dagger} D_{i} \psi+\frac{e}{2 m c} \psi^{\dagger} B_{12} \psi\right. \\
& \left.+\lambda_{1}\left(\phi^{\dagger} \phi \phi^{\dagger} \phi\right)+\lambda_{2}\left(\phi^{\dagger} \phi \psi^{\dagger} \psi\right)+\lambda_{3}\left(\phi \phi^{\dagger} \psi \psi^{\dagger}\right)+\lambda_{4}\left(\psi^{\dagger} \psi \psi^{\dagger} \psi\right)\right) .
\end{aligned}
$$

This action is the simplest generalization to noncommutative space of the one studied in Ref. [10]. Notice that terms of the potential proportional to $\lambda_{2}$ and $\lambda_{3}$ would be equivalent to each other in ordinary space and that the term proportional to $\lambda_{4}$ would be identically zero due to the anticommuting character of the fermion fields. We have chosen a particular sign for the Pauli interaction (corresponding to a "down" spinor) which coincides with the one in [10].

In a nonrelativistic setting the action of bosons and fermions are almost identical if it were not for the presence of the Pauli term for fermions. As shown below, the supersymmetry variation of this last term is easily compensated by the Chern-Simons term.

We will calculate the variation of the action under the following supersymmetry transformation:

$$
\delta_{1} \phi=\sqrt{2 m} \eta_{1}^{\dagger} \psi, \quad \delta_{1} \psi=-\sqrt{2 m} \eta_{1} \phi, \quad \delta_{1} A=0, \quad \delta_{1} A^{0}=\frac{e}{\sqrt{2 m} c \kappa}\left(\eta_{1} \phi \psi^{\dagger}-\eta_{1}^{\dagger} \psi \phi^{\dagger}\right) .
$$

It is trivial to show that,

$$
\begin{aligned}
& \delta_{1} \int\left(-\frac{\kappa}{4 i c}\left(\left(\partial_{t} A\right)_{+} A_{-}-\left(\partial_{t} A\right)_{-} A_{+}\right)+i \phi^{\dagger} \partial_{t} \phi+i \psi^{\dagger} \partial_{t} \psi\right) d^{3} x=0, \\
& \delta_{1} \int\left(\frac{1}{2 m}(D \phi)^{\dagger}(D \phi)+(D \psi)^{\dagger}(D \psi)\right) d^{3} x=0, \quad \delta_{1} \int\left(-A_{0} \kappa B_{12}+\frac{e}{2 m c} \psi^{\dagger} B_{12} \psi\right) d^{3} x=0 .
\end{aligned}
$$

Then the variation of the action reduces to

$$
\begin{aligned}
\delta_{1} S= & \sqrt{2 m} \int d^{3} x\left(\eta _ { 1 } \left[\left(-\frac{e^{2}}{2 m c \kappa}-2 \lambda_{1}-\lambda_{3}+\lambda_{2}\right) \phi^{\dagger} \phi \psi^{\dagger} \phi\right.\right. \\
& \left.\left.+\left(\frac{e^{2}}{2 m c \kappa}-\lambda_{3}+2 \lambda_{4}-\lambda_{2}\right) \psi^{\dagger} \phi \psi^{\dagger} \psi\right]+ \text { h.c. }\right) .
\end{aligned}
$$


So the transformations in Eq. (14) correspond to a symmetry of the action if the following relations are satisfied:

$$
\frac{e^{2}}{2 m c \kappa}+2 \lambda_{1}+\lambda_{3}-\lambda_{2}=0, \quad \frac{e^{2}}{2 m c \kappa}-\lambda_{3}+2 \lambda_{4}-\lambda_{2}=0
$$

While the first condition coincides with the one arising in ordinary space, the second one is peculiar to noncommutative space. It originates from the second term in Eq. (14) which is automatically zero in ordinary space due to the Grassman character of $\psi$ and $\psi^{\dagger}$.

The fact that is less evident is the existence of a second supersymmetry. Let us examine the variation of the action under the following transformation:

$$
\begin{aligned}
& \delta_{2} \phi=\frac{i}{\sqrt{2 m}} \eta_{2}^{\dagger} D_{+} \psi, \quad \delta_{2} \psi=-\frac{i}{\sqrt{2 m}} \eta_{2} D_{-} \phi, \\
& \delta_{2} A^{+}=\frac{2 e}{\sqrt{2 m} \kappa} \eta_{2} \phi \psi^{\dagger}, \quad \delta_{2} A^{-}=-\frac{2 e}{\sqrt{2 m}} \eta_{2}^{\dagger} \psi \phi^{\dagger}, \\
& \delta_{2} A^{0}=\frac{i e}{(2 m)^{3 / 2} c \kappa}\left(\eta_{2} \phi\left(D_{+} \psi\right)^{\dagger}+\eta_{2}^{\dagger}\left(D_{+} \psi\right) \phi^{\dagger}\right) .
\end{aligned}
$$

Again it is easy to show the invariance of the kinetic terms

$$
\delta \int\left(-\frac{\kappa}{4 i c}\left(\left(\partial_{t} A\right)_{+} A_{-}-\left(\partial_{t} A\right)_{-} A_{+}\right)+i \phi^{\dagger} \partial_{t} \phi+i \psi^{\dagger} \partial_{t} \psi\right) d^{3} x=0 .
$$

After some algebra, one can see that the variation of the remaining part of the action can be written as

$$
\delta_{2} S=\delta_{2}^{(1)} S+\delta_{2}^{(3)} S
$$

where $\delta_{2}^{(1)} S$ is linear in fermion fields and $\delta_{2}^{(3)} S$ is cubic:

$$
\begin{aligned}
\delta_{2}^{(1)} S= & \frac{i}{\sqrt{2 m}} \eta_{2} \int d^{3} x\left(\psi^{\dagger}\left(\frac{e^{2}}{2 m c \kappa} 2 \phi\left(D_{+} \phi\right)^{\dagger} \phi+\phi \phi^{\dagger}\left(D_{-} \phi\right)\right)\right. \\
& \left.-2 \lambda_{1}\left(\phi\left(D_{+} \phi\right)^{\dagger} \phi+\phi \phi^{\dagger}\left(D_{-} \phi\right)+\left(D_{-} \phi\right) \phi^{\dagger} \phi\right)+\lambda_{2}\left(D_{-} \phi\right) \phi^{\dagger} \phi-\lambda_{3}\left(D_{-} \phi\right) \phi \phi^{\dagger}\right)+ \text { h.c., } \\
\delta_{2}^{(3)} S= & \frac{i}{\sqrt{2 m}} \eta_{2} \int\left(d ^ { 3 } x \psi ^ { \dagger } \left(\frac{e^{2}}{2 m c \kappa}\left(\phi\left(D_{+} \psi\right)^{\dagger} \psi+2 \psi\left(D_{+} \psi\right)^{\dagger} \phi\right)+\lambda_{2} \psi\left(D_{+} \psi\right)^{\dagger} \phi+\lambda_{3} \phi\left(D_{+} \psi\right)^{\dagger} \psi\right.\right. \\
& \left.\left.-2 \lambda_{4}\left(\phi\left(D_{+} \psi\right)^{\dagger} \psi+\phi \psi^{\dagger}\left(D_{-} \psi\right)+\psi\left(D_{+} \psi\right)^{\dagger} \phi\right)\right)\right)+ \text { h.c. }
\end{aligned}
$$

From Eq. (21), we obtain

$$
\frac{e^{2}}{m c \kappa}-2 \lambda_{1}=0, \quad \frac{e^{2}}{2 m c \kappa}-2 \lambda_{1}-\lambda_{3}=0, \quad-2 \lambda_{1}+\lambda_{2}=0,
$$

while from Eq. (22) we get

$$
\frac{e^{2}}{2 m c \kappa}+\lambda_{3}-2 \lambda_{4}=0, \quad \lambda_{4}=0, \quad-\frac{e^{2}}{m c \kappa}+\lambda_{2}-2 \lambda_{4}=0 .
$$

The solution for this system is

$$
\lambda_{1}=\frac{e^{2}}{2 m c \kappa}, \quad \lambda_{2}=\frac{e^{2}}{m c \kappa}, \quad \lambda_{3}=-\frac{e^{2}}{2 m c \kappa}, \quad \lambda_{4}=0,
$$

which also satisfies the system of Eqs. (17). 
The results in ordinary space, namely

$$
\frac{e^{2}}{2 m c \kappa}+2 \lambda_{1 c}-\lambda_{2 c}=0, \quad \lambda_{1 c}=\frac{e^{2}}{2 m c \kappa},
$$

can be recovered by noticing that the potential in ordinary space becomes

$$
V=\lambda_{1} \phi^{\dagger} \phi \phi^{\dagger} \phi+\left(\lambda_{2}-\lambda_{3}\right) \phi \phi^{\dagger} \psi \psi^{\dagger},
$$

so that $\lambda_{2 c}=\lambda_{2}-\lambda_{3}$ can be identified. After this identification, the ordinary space results follow.

The relation of coupling constants which make the model $N=2$ supersymmetric is connected to the BPS point. This can be most easily seen by writing the Hamiltonian of the model as

$$
H=\int d^{2} x\left(\frac{1}{2 m}\left(D_{i} \phi\right)^{\dagger} D_{i} \phi+\frac{1}{2 m}\left(D_{i} \psi\right)^{\dagger} D_{i} \psi-\frac{e}{2 m} \psi^{\dagger} B \psi+V(\phi, \psi)\right),
$$

where

$$
V[\phi, \psi]=-\lambda_{1} \phi^{\dagger} \phi \phi^{\dagger} \phi-\lambda_{2} \phi^{\dagger} \phi \psi^{\dagger} \psi-\lambda_{3} \phi \phi^{\dagger} \psi \psi^{\dagger}-\lambda_{4} \psi^{\dagger} \psi \psi^{\dagger} \psi
$$

and

$$
B=-\frac{e}{\kappa}\left(\phi \phi^{\dagger}-\psi \psi^{\dagger}\right)
$$

Using the identity

$$
\left(D_{i} \phi\right)^{\dagger} D_{i} \phi=\left(D_{ \pm} \phi\right)^{\dagger} D_{ \pm} \phi \pm e \phi^{\dagger} B \phi,
$$

the Hamiltonian can be re-written (up to surface terms) as

$$
\begin{aligned}
H= & \int d^{2} x\left(\frac{1}{2 m}\left(D_{ \pm} \phi\right)^{\dagger} D_{ \pm} \phi+\frac{1}{2 m}\left(D_{ \pm} \psi\right)^{\dagger} D_{ \pm} \psi-\left(\lambda_{1} \pm \frac{e^{2}}{2 m \kappa}\right) \phi^{\dagger} \phi \phi^{\dagger} \phi-\lambda_{2} \phi^{\dagger} \phi \psi^{\dagger} \psi\right. \\
& \left.+\left(-\lambda_{3} \pm \frac{e^{2}}{m \kappa}-\frac{e^{2}}{2 m \kappa}\right) \phi \phi^{\dagger} \psi \psi^{\dagger}+\left(-\lambda_{4} \pm \frac{e^{2}}{2 m \kappa}-\frac{e^{2}}{2 m \kappa}\right) \psi^{\dagger} \psi \psi^{\dagger} \psi\right) .
\end{aligned}
$$

In the bosonic sector of the theory, we then have

$$
H=\int d^{2} x\left(\frac{1}{2 m}\left(D_{ \pm} \phi\right)^{\dagger} D_{ \pm} \phi-\left(\lambda_{1} \pm \frac{e^{2}}{2 m \kappa}\right) \phi^{\dagger} \phi \phi^{\dagger} \phi\right) .
$$

Thus, taking the lower sign,

$$
H=\int d^{2} x \frac{1}{2 m}\left(D_{ \pm} \phi\right)^{\dagger} D_{ \pm} \phi
$$

leads to the BPS equation

$$
D_{-} \phi=0 \text {. }
$$

The particular choice of sign in Eq. (33) which leads to the "antiselfdual" Eq. (35) is related to our earlier choice of sign for the Pauli interaction. Changing this sign and redefining the SUSY transformations accordingly leads to the "selfdual" BPS equation.

In ordinary space, the choice that makes the model $N=2$ supersymmetry invariant allows to write the full Hamiltonian (i.e., bosons + fermions) as a sum of squares [10], just the first two terms in (32). Nevertheless, in NC space, one obtains

$$
H=\int d^{2} x\left(\frac{1}{2 m}\left(D_{-} \phi\right)^{\dagger} D_{-} \phi+\frac{1}{2 m}\left(D_{-} \psi\right)^{\dagger} D_{-} \psi-\frac{e^{2}}{m \kappa} \phi^{\dagger} \phi \psi^{\dagger} \psi-\frac{e^{2}}{m \kappa} \phi \phi^{\dagger} \psi \psi^{\dagger}-\frac{e^{2}}{m \kappa} \psi^{\dagger} \psi \psi^{\dagger} \psi\right) .
$$


The extra terms cancels in the commutative limit.

In order to write down the supersymmetry algebra, we start by defining Poisson brackets. Calling $F, G$ the supersymmetry charges or their Hermitian conjugates, we have

$$
\{F, G\}=i \sum_{j} \int d^{2} x\left(\frac{\delta F}{\delta \Omega_{j}(x, t)} \frac{\delta G}{\delta \Pi_{j}(x, t)}-(-1)^{f_{j}} \frac{\delta F}{\delta \Pi_{j}(x, t)} \frac{\delta G}{\delta \Omega_{j}(x, t)}\right),
$$

with

$$
\Omega=\left(\phi, \psi, \sqrt{\frac{\kappa}{2}} A_{+}\right), \quad \Pi=\left(i \phi^{\dagger}, i \psi^{\dagger}, i \sqrt{\frac{\kappa}{2}} A_{-}\right), \quad f=(0,1,0) .
$$

Using Noether's theorem, the supersymmetric transformations (14) and (18) lead to the charges $Q_{1}$ and $Q_{2}$,

$$
\begin{aligned}
& Q_{1}=i \sqrt{2 m} \int d^{2} x \phi^{\dagger} \psi, \\
& Q_{2}=\frac{1}{\sqrt{2 m}} \int d^{2} x\left(D_{-} \phi\right)^{\dagger} \psi .
\end{aligned}
$$

Using (37) one gets for the $Q_{1}$ bracket

$$
\left\{Q_{1}, Q_{1}^{\dagger}\right\}=2 m \int d^{2} x\left(\phi^{\dagger} \phi+\psi^{\dagger} \psi\right) \equiv 2 M,
$$

where we have introduced the total mass $M$.

Concerning the $Q_{2}$ bracket, one has

$$
\left\{Q_{2}, Q_{2}^{\dagger}\right\}=\frac{1}{2 m} \int d^{2} z\left(\left(D_{+} \psi\right)^{\dagger}(z) D_{+} \psi(z)+\left(D_{-} \phi\right)^{\dagger}(z) D_{-} \phi(z)-\frac{2 e^{2}}{\kappa} \phi^{\dagger} \psi^{\dagger} \psi \phi\right) .
$$

Now, identity (31) allows us to rewrite Eq. (42) in the form

$$
\left\{Q_{2}, Q_{2}^{\dagger}\right\}=\frac{1}{2 m} \int d^{2} z\left(\left(D_{i} \psi\right)^{\dagger}(z) D_{i} \psi(z)+\left(D_{i} \phi\right)^{\dagger}(z) D_{i} \phi(z)-\frac{2 e^{2}}{\kappa} \phi^{\dagger} \psi^{\dagger} \psi \phi+e \phi^{\dagger} B \phi-e \psi^{\dagger} B \psi\right),
$$

which, after using the Gauss law (Eq. (30)) becomes

$$
\left\{Q_{2}, Q_{2}^{\dagger}\right\}=H \text {. }
$$

Finally, the only nonvanishing remaining bracket gives

$$
\left\{Q_{1}, Q_{2}^{\dagger}\right\}=\frac{1}{2 i} \int d^{2} x\left(\phi^{\dagger} D_{-} \phi-\left(D_{-} \phi\right)^{\dagger} \phi+\psi^{\dagger} D_{-} \psi-\left(D_{-} \psi\right)^{\dagger} \psi\right)=P_{-},
$$

being $P_{i}$ the momentum.

As in the ordinary space case notice that the configurations of fields $(\phi, \psi)$ such that

$$
D_{-} \phi=0, \quad \psi=0
$$

is left invariant by the supersymmetric transformation Eqs. (18).

In summary, we have been able to show that a $N=2$ supersymmetric extension of model for nonrelativistic matter interacting with Chern-Simons gauge fields can be built. This was achieved for a particular relation of coupling constants given in Eq. (25), which in the commutative space limit $\theta=0$ reduces to the ordinary space result [10]. In NC space, even for a $U(1)$ gauge group one has different possibilities to couple gauge and matter fields according to the choice of the covariant derivative (in the fundamental, the antifundamental or the adjoint 
representation) and this can in principle lead to different sets of BPS equations and their corresponding solutions. Also, since the NC extension of the Jackiw-Pi model [4] could be of relevance in connection with the physics of the quantum Hall effect, the case of nonrelativistic matter interacting with non-Abelian gauge fields would be of much interest. In this respect, we believe that our results can be extended to the case of a $U(N)$ group as it was done in ordinary space for the $S U(N)$ case [16]. We expect to report on these issues in the future.

\section{Acknowledgements}

We acknowledge financial support from a CAPES-SECyT grant \#59/03. F.A.S. is partially supported by a CICBA grant. G.S.L. thanks the ICTP, Trieste, were part of this work has been done and Carlos Nuñez for interesting discussions.

\section{References}

[1] M.R. Douglas, N.A. Nekrasov, Rev. Mod. Phys. 73 (2001) 977, hep-th/0106048; R.J. Szabo, Phys. Rep. 378 (2003) 207, hep-th/0109162.

[2] L. Susskind, hep-th/0101029.

[3] For more recent discussions see D. Berenstein, Phys. Rev. D 71 (2005) 085001, hep-th/0409115; A. Ghodsi, A.E. Mosaffa, O. Saremi, M.M. Sheikh-Jabbari, hep-th/0505129.

[4] R. Jackiw, S.Y. Pi, Phys. Rev. Lett. 64 (1990) 2969;

R. Jackiw, S.Y. Pi, Phys. Rev. D 42 (1990) 3500; R. Jackiw, S.Y. Pi, Phys. Rev. D 48 (1993) 3929, Erratum.

[5] G.S. Lozano, E.F. Moreno, F.A. Schaposnik, JHEP 0102 (2001) 036, hep-th/0012266.

[6] C.R. Hagen, Phys. Rev. D 31 (1985) 848.

[7] O. Bergman, G. Lozano, Ann. Phys. 229 (1994) 416, hep-th/9302116; D.Z. Freedman, G. Lozano, N. Rius, Phys. Rev. D 49 (1994) 1054, hep-th/9306117.

[8] P.A. Horvathy, L. Martina, P.C. Stichel, Phys. Lett. B 615 (2005) 87, hep-th/0412090; P.A. Horvathy, L. Martina, P.C. Stichel, hep-th/0411139; P.A. Horvathy, L. Martina, P.C. Stichel, Nucl. Phys. B 673 (2003) 301, hep-th/0306228; P.A. Horvathy, L. Martina, P.C. Stichel, Phys. Lett. B 564 (2003) 149, hep-th/0304215.

[9] D. Bak, K. Lee, J.-H. Park, Phys. Rev. D 63 (2001) 125010; D. Bak, Phys. Lett. B 495 (2000) 251.

[10] M. Leblanc, G. Lozano, H. Min, Ann. Phys. 219 (1992) 328, hep-th/9206039.

[11] E. Witten, D.I. Olive, Phys. Lett. B 78 (1978) 97.

[12] C.K. Lee, K.M. Lee, E.J. Weinberg, Phys. Lett. B 243 (1990) 105.

[13] Z. Hlousek, D. Spector, Nucl. Phys. B 370 (1992) 143; Z. Hlousek, D. Spector, Phys. Lett. B 283 (1992) 75.

[14] J.D. Edelstein, C. Nunez, F. Schaposnik, Phys. Lett. B 329 (1994) 39, hep-th/9311055.

[15] R. Puzalowski, Acta Phys. Austriaca 50 (1978) 45.

[16] D. Bak, R. Jackiw, S.Y. Pi, Phys. Rev. D 49 (1994) 6778, hep-th/9402057. 